\title{
Pathologic changes and dysfunctions of astrocytes in the complex rat AD model of ovariectomy combined with D-galactose injection
}

\author{
Tao YQ, Liang GB \\ Department of Neurosurgery, General Hospital of Shenyang Military Region,WenhuaRoad, ShenheDistrict, \\ Shenyang, LiaoningProvince, China. liangguobiao70@163.com
}

\begin{abstract}
Objective: To study pathogenesis of astrocytes in the SD model. Method: The male adult SD rats were divided into 3 groups at random with 10 for each group to study pathologic changes and dysfunctions of astrocytes in the model with 3 weeks and 6 weeks of observation time.

Results: Compared with the control group and the 3w O+D group, the incubation period for the rats of the $6 \mathrm{w}$ $O+D$ group to reach the platform is significantly prolonged in the water maze behavioral experiment; the times of passing through the platform and the duration for staying in the target quadrant are significantly decreased but there is no statistical difference between the control group and the 3w O+D group; compared with rats of the control group and the $3 \mathrm{w} \mathrm{O}+\mathrm{D}$ group, the rats of the 6w O+D group show signs of significant density decrease and AChE activity decrease of cholinergic fiber terminals in the hippocampal region, but there is no statistical difference between the control group and the $3 w \mathrm{O}+\mathrm{D}$ group; compared with control group, the GFAP level of in the hippocampal region of the rats of the $3 \mathrm{w} O+D$ group and the $6 \mathrm{w} \mathrm{O+D} \mathrm{group} \mathrm{significantly} \mathrm{increases} \mathrm{but} \mathrm{there} \mathrm{is} \mathrm{no}$ statistical difference between the two groups; the hippocampi and some areas of the cerebral cortices of the rats of the $6 \mathrm{~W}$ O+D group show signs of HRP leakage in the peripheral region of blood vessels. However, HRP reaction products of the control group are distributed within the blood vessels and show no signs of significant leakage. Conclusion: OVX combined with injection of D-gal can serve as a good animal model simulating AD disease. Astrocytes play an important role in occurrence and progression of pathological changes of the model (Tab. 6, Fig. 6, Ref. 30). Text in PDF www.elis.sk.

Key words: astrocytes, ovariectomy, D-galactose, complex AD model, pathologic change, dysfunctions.
\end{abstract}

Astrocytes are the most abundant and most widely distributed cells in the central nervous system. They play an important role in adjusting synaptic network formation, electrical activities of neurons and completeness of blood brain barrier (1). Previous researches show that neurons and capillaries and astrocytes can constitute «nerve-colloid corpuscle» and «colloid -blood vessel corpuscle». These corpuscles play an important role in maintaining stability of the structure and functions of the central nervous system (2). Astrocytes are involved in the pathological process of multiple degenerative diseases of the nervous system, such as multiple sclerosis, Parkinson's disease, Alzheimer Disease (AD), etc. (3). In the vicinity of intracerebral extracellular $A \beta$ plaques of $\mathrm{AD}$ patients and in animal models (characteristic neuropathological change), a large number of astrocytes are activated (4). In addition, astrocytes are also involved in formation of another characteristic neuropathological change, NFT (5). The above research background indicates that activated astrocytes may play a vital role in the process of $\mathrm{AD}$ invasion. However, morphologi-

Department of Neurosurgery, General Hospital of Shenyang Military Region, Wenhua Road, Shenhe District, Shenyang, Liaoning Province, China Address for correspondence: GB Liang, Department of Neurosurgery, General Hospital of Shenyang Military Region, No. 83, Wenhua Road, Shenhe District, Shenyang, Liaoning Province, China. Zip code: 110840 cal and biochemical changes of astrocytes and the correlation between such changes and cholinergic defect and dysmnesia are still worth further study in different stages of AD invasion (6). Endogenous estrogen deficiency and oxidative stress are two critical factors closely associated with occurrence and progression of $\mathrm{AD}$ (7). Intraperitoneal injection of D-gal can cause damage to cognition of OVX rats, loss of basal forebrain cholinergic neurons, appearance of $\mathrm{A} \beta$ positive cells in the hippocampal region and similar NFT changes (8). OVX combined with long-term injection of $\mathrm{D}$-gal can properly simulate $\mathrm{AD}$ pathologic changes and is a new complex AD animal model. Based on the fact that endogenous estrogen deficiency and oxidative stress can cause activation of astrocytes, we infer that the morphological and biochemical changes of astrocytes may be involved in development of $\mathrm{AD}$ disease.

\section{Material and methods}

\section{Material}

The experimental animals were 30 female adult SD rats weighing 200-220 g, purchased from SHANGHAI SLAC LABORATORY ANIMAL CO. LTD. All animals were fed and watered ad libitum, raised in a warm $\left(18-22{ }^{\circ} \mathrm{C}\right)$, quiet, natural circadian rhythm sunlight environment and weighed once every week. 
The D-gal was purchased from U.S. Fermentas Company; anti-GFAP murine monoclonal antibody was purchased from U.S. Sigma Company; HRP conjugated anti-goat mouse IgG was purchased from U.S. SantaCruz Company; biotinylated anti-goat mouse IgG was purchased from U.S. Amresco Company; SABC compound was purchased from Hangzhou Biowish biotechnology Co. Ltd.; DAB was purchased from Shanghai Jianglai Biotechnology Co., Ltd.; acetylthiocholine iodide was purchased from Shanghai Boyan Biotechnology Co., Ltd.; GSH and AChE kits were purchased from Shanghai Yanjing Biotechnology Co., Ltd.; HRP was purchased from Beijing CoWin Bioscience Co., Ltd.

\section{Grouping of animals}

The rats were divided into 3 groups with 10 for each group 1 week after acclimatization.

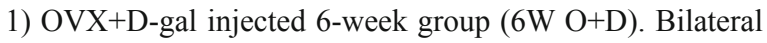
OVX was performed in the rats. Intraperitoneal injection of D-gal was injected at dose $60 \mathrm{mg} / \mathrm{kg} / \mathrm{d} 2$ days after operation and lasted for 6 weeks. 2) OVX+D-gal injected 3-week group (3W O+D). Bilateral OVX was performed for the rats at the end of the 3rd week. Intraperitoneal injection of D-gal was injected at dose 60 $\mathrm{mg} / \mathrm{kg} / \mathrm{d} 2$ days after operation and lasted for 3 weeks; 3 ) the rats of the control group were intraperitoneally injected with normal saline $5 \mathrm{ml} / \mathrm{d}$ for consecutive 6 weeks (9).

\section{Water maze}

We evaluated the effect of OVX and D-gal on spatial memory ability through the water maze behavior test. The time for each rat reaching the platform was recorded through digital camera and a computer system. The test lasted for 5 consecutive days with 3 times for each day; the platform was removed and an exploration test was conducted on the 5th day. Each rat swam for 90 seconds autonomously. The time of stay in the front platform quadrant and times of passing the position where the front platform was located were recorded (10).

\section{Tissue preparation}

The rats were anesthetized with pentobarbital sodium (100 $\mathrm{mg} / \mathrm{kg}$, intraperitoneal injection) following the behavior test. The brain was taken for use in: 1) detection of biochemical indexes: The brain was taken freshly. The hippocampus was isolated from the brain and stored at $-80^{\circ} \mathrm{C}$ for later use; 2) AChE histochemical staining: the rats were perfused with $4 \%$ paraformaldehyde. Then the brain tissue containing the hippocampus was placed in a $4 \%$ paraformaldehyde solution for fixation at $4{ }^{\circ} \mathrm{C}$ overnight, placed in $0.1 \mathrm{M}$ phosphate buffer containing $30 \%$ of sucrose at $4{ }^{\circ} \mathrm{C}$ overnight, sectioned into $30 \mu \mathrm{m}$-thick coronary slices with a freezing microtome. 3) GFAP immunohistochemical staining: The brain tissue containing hippocampus was dehydrated in gradient alcohol, embedded in paraffin, sectioned into $5 \mu \mathrm{m}$-thick coronary slices. 2 slices were taken from every 10 slices. 2 sets of slices were chosen for backup. HE staining was performed for one set and immunohistochemical staining was performed for the other set. 4) Western-blotting: The brain was taken freshly. The hippocampus was isolated from the brain. Total protein was extracted in regular homogenate. It was stored at $-80^{\circ} \mathrm{C}$ for later use.

\section{Western-blotting detection}

The hippocampus tissue specimen was electrophoresed at a constant voltage of $15 \mathrm{~V}$ in $5 \%$ spacer gel and $10 \%$ separation gel, transferred for $1 \mathrm{~h}$ at $300 \mathrm{~mA}$, blocked in $5 \%$ skim milk powder at $4{ }^{\circ} \mathrm{C}$ overnight, incubated in the anti-GFAP murine monoclonal antibody (chemicon, $1: 500$ ) humidified box at $37^{\circ} \mathrm{C}$ for $1 \mathrm{~h}$, washed with TBST for $10 \mathrm{~min} \times 3$ times, incubated in the HRP conjugated rabbit antimouse IgG (Vector, $1: 150$ ) humidified box at $37^{\circ} \mathrm{C}$ for $1 \mathrm{~h}$, washed with TBST for $10 \mathrm{~min} \times 3$ times, exposed in a darkroom following addition of ECL, developed and fixed. The process was repeated for 3 times. The absorbance value was determined with the Leica full-automatic image analyzer. The ratio of GFAP optical density (OD) to $\beta$-actin OD was regarded as GFAP relative optical density value.

\section{GSH detection}

The hippocampal tissue was placed in a buffer solution containing $0.01 \mathrm{M}$ Tris- $\mathrm{HCl}(\mathrm{pH} 7.4), 0.01 \mathrm{M}$ sucrose and $0.1 \mathrm{mM}$ EDTA and homogenized in a homogenizer at 2000 revolutions per second for $30 \mathrm{~s}$. Then it was homogenized at 4000 revolutions per minute and centrifuged for $15 \mathrm{~min}$ at $4{ }^{\circ} \mathrm{C}$. The supernatant was collected and detected in accordance with the GSH kit from Nanjing Jiancheng Biological Research Laboratory. The optical density value of the reaction product was detected with a UV-240 spectrophotometer.

\section{AChE activity detection}

The hippocampal tissue was placed in a buffer solution containing $0.01 \mathrm{M}$ Tris- $\mathrm{HCl}(\mathrm{pH} 7.4), 0.01 \mathrm{M}$ sucrose and $0.1 \mathrm{mM}$ EDTA and homogenized in a homogenizer at 2000 revolutions per second for $30 \mathrm{~s}$. Then it was homogenized at 3500 revolutions per minute and centrifuged for $10 \mathrm{~min}$ at $4{ }^{\circ} \mathrm{C}$. The supernatant was collected and detected in accordance with the instructions for AChE kit. And the optical density value of the reaction product was detected with an UV-240 spectrophotometer.

\section{Immunohistochemical staining of GFAP}

The paraffin sections of hippocampus were dewaxed to water in gradient alcohol, placed in a $3 \%$ hydrogen peroxide for inactivation of endogenous peroxidase for $15 \mathrm{~min}$, washed with PBS for $\mathrm{x} 3$ times, antigen microwave repaired in the citrate buffer solution at $92-98{ }^{\circ} \mathrm{C}$ for $20 \mathrm{~min}$, naturally cooled to room temperature, blocked in a $5 \%$ normal goat blocking serum for $30 \mathrm{~min}$, incubated in anti-GFAP murine monoclonal antibody (Chemicon, $1: 500$ ) wet box at $37^{\circ} \mathrm{C}$ for $1 \mathrm{~h}$, washed with PBS for $5 \mathrm{~min} \mathrm{x} 3$ times, incubated in a biotinylated goat anti-mouse $\operatorname{IgG}$ (Vector, $1: 150$ ) wet box at $37^{\circ} \mathrm{C}$ for $40 \mathrm{~min}$, washed with PBS for $5 \mathrm{~min}$ $\mathrm{x} 3$ times, incubated in a SABC compound wet box at $37^{\circ} \mathrm{C}$ for 20 min, washed with PBS FOR 5 min $x 3$ times, developed in DAB at room temperature, the reaction was controlled under microscope, dehydrated regularly, clarified, slide mounted, observed and photographed under a light microscope. 


\section{Histochemical staining of AChE}

The frozen sections were washed with $0.1 \mathrm{M}$ acetic acid buffer solution ( $\mathrm{pH}$ 6.0) for 5 min $\mathrm{x} 3$ times, placed in the incubation buffer at room temperature for $30 \mathrm{~min}$, sufficiently rinsed with 0.1 $\mathrm{M}$ acetic acid buffer solution ( $\mathrm{pH}$ 6.0), incubated for $2 \mathrm{~min}$ after addition of $1 \%$ ammonium sulfide, sufficiently rinsed with 0.1 $\mathrm{M}$ sodium nitrate, incubated for $1 \mathrm{~min}$ following addition of 0.1 $\%$ silver nitrate, sufficiently rinsed with $0.1 \mathrm{M}$ sodium nitrate, dehydrated, mounted and observed under microscope. 4 sections were chosen from the same site of hippocampus of each rat and statistical analysis was performed for the average optical density of AChE positive fiber with Leica Qwin full-automatic image analysis system.

\section{Observation by electron microscope}

The rat brain was sectioned into $80 \mu \mathrm{m}$-thick serial coronary slices with a vibratome and collected in $0.1 \mathrm{~mol} / \mathrm{L} \mathrm{PB}$ at $\mathrm{pH}$ 7.4. Hippocampal CAl region and cerebral cortex were taken. Each piece was approximately $1 \mathrm{~mm} \times 1 \mathrm{~mm}$, fixed in $1 \%$ osmic acid for $1 \mathrm{~h}$, dehydrated in gradient acetone, dipped in $1: 4$ and $1: 2$ gradient Epon618, embedded on silicified slides with Epon618 plate, sectioned with the model LKB-5 ultrathin microtome, double stained with uranyl acetate and lead citrate, observed, counted and photographed under a transmission electron microscope. Changes of ultrastructure of neurons in hippocampus and cerebral cortex regions were observed under the electron microscope. 3 copper wire meshes (with diameter of $0.3 \mathrm{~mm}, 200$ meshes) were observed for each rat. Each mesh was shifted parallel from left to right to observe changes of synapses, lipofuscin etc. Counting and photographing were performed.

\section{HRP test}

HRP was injected into the heart of the rat $(0.3 \mathrm{mg} / \mathrm{g}$ body weight $/ 0.1 \mathrm{ml}$ normal saline). The rat was decollated and its brain was separated out after $5 \mathrm{~min}$. The brain was placed in $0.1 \mathrm{M}$ cacodylic acid sodium salt trihydrate buffer solution containing 3\% glutaraldehyde $(\mathrm{pH} 7.3)$ at $4{ }^{\circ} \mathrm{C}$ for $3 \mathrm{~h}$, then washed with the same buffer solution for $12 \mathrm{~h}$, and sectioned to $50 \mu \mathrm{m}$-thick slices with a vibratome. Subsequently, the sections were placed in the $0.05 \mathrm{M}$ Tris- $\mathrm{HCl}$ buffer solution containing $0.05 \%$ 3,3'-diaminodiphenyl and $0.01 \% \mathrm{H}_{2} \mathrm{O}_{2}$ for incubation at room temperature for $30 \mathrm{~min}$.

\section{Statistical analysis}

Experimental data in ethological and morphological detection was expressed as mean \pm standard deviation $( \pm \mathrm{s})$. The SPSS 15.0 statistical analysis software was applied for variance analysis or rank sum test. It is defined that $\mathrm{p}<0.05$ indicates a significant level.

\section{Results}

Retrogression of cognitive function and cholinergic loss of $O V X+D$-gal rats

All tested rats showed no significant health problem, such as weight reduction, skin damage or appetite loss etc. During the whole training process, the latency period for rats of the $6 \mathrm{w} \mathrm{O}+\mathrm{D}$ group to reach the platform is significantly longer than that of the control group and the rats of the $3 \mathrm{w} \mathrm{O}+\mathrm{D}$ group whereas there is no statistical difference in latency periods between the control group rats and the $3 \mathrm{w} \mathrm{O}+\mathrm{D}$ group rats (Fig. 1A).

The platform was removed and an exploration experiment was performed at the 5 th day of training. The swimming time of rats of the control group and the $3 \mathrm{w} \mathrm{O}+\mathrm{D}$ group was within the quadrant where the platform was located. There was no significant difference (Fig. 1B).

The swimming time of the $6 \mathrm{w} \mathrm{O}+\mathrm{D}$ group was uniformly distributed in 4 quadrants. The result of the experiment of platform passing through and the result of this experiment are consistent. The times of the control group rats and the $3 \mathrm{w} \mathrm{O}+\mathrm{D}$ rats passing the platform is significantly more than that of the $6 \mathrm{w} \mathrm{O+D}$ group of rats. There is no significant difference in times of passing through between the control group and the $3 \mathrm{w} \mathrm{O}+\mathrm{D}$ group (Fig. 1C) (Tab. 1).
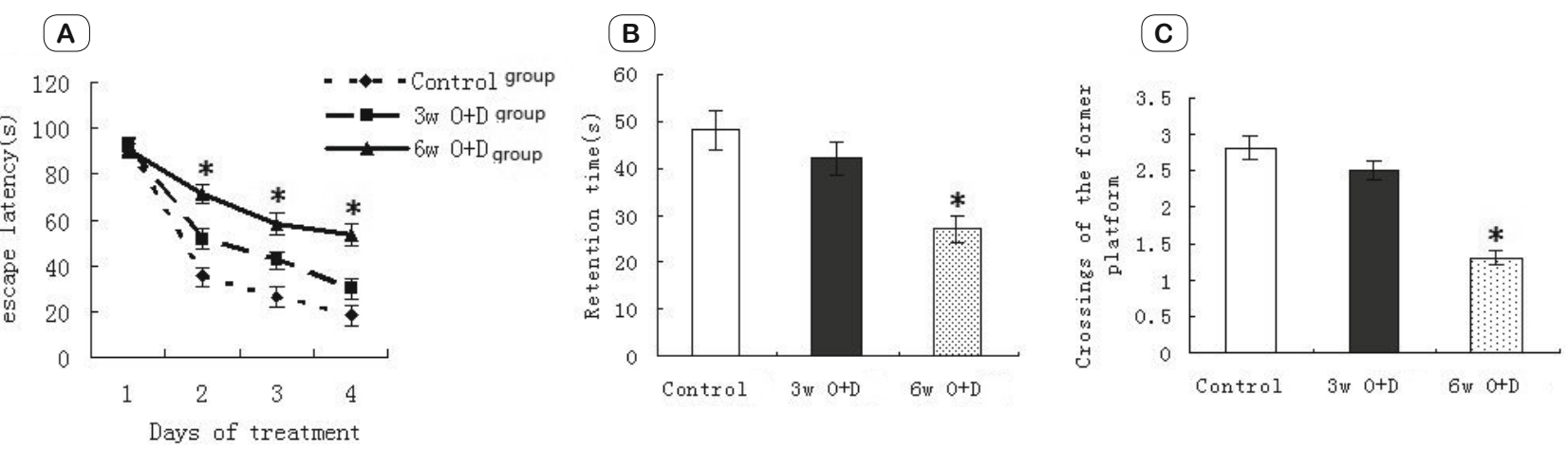

Tab. 1.Test result of rats` cognitive competence.

\begin{tabular}{ll}
\hline Control Group & Control Group \\
\hline ww O+D Group & $3 \mathrm{w} \mathrm{O}+\mathrm{D}$ Group \\
\hline $\begin{array}{l}\text { A }- \text { test of the platform latency period on the 4th day. B }- \text { Time for swimming within the platform quadrant on the 5th day; C }- \text { Test result of time for platform passing } \\
\text { through. Comparison with the negative control indicates that } * \mathrm{p}<0.05 .\end{array}$
\end{tabular}


(A)
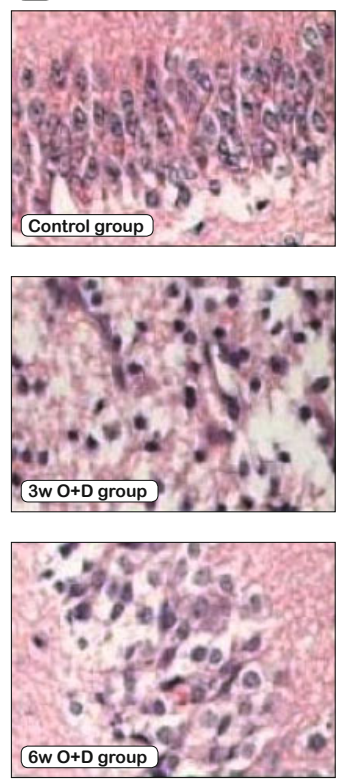

(B)

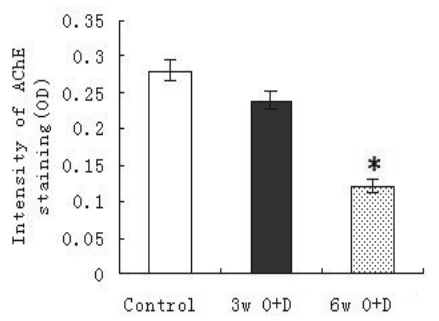

C

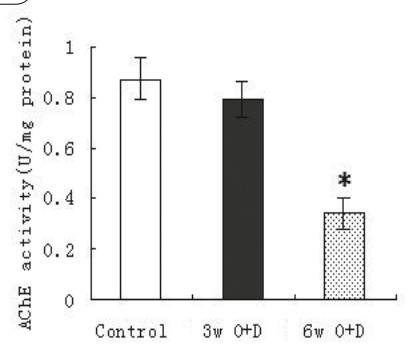

Tab. 2. Conditions of the cholinergic fiber endings in the hippocampal region.

\begin{tabular}{ll}
\hline Control Group & Control Group \\
\hline $3 w \mathrm{O}+\mathrm{D}$ Group & $3 w \mathrm{O}+\mathrm{D}$ Group \\
\hline 6w O+D Group & $6 w \mathrm{O}+\mathrm{D}$ Group \\
\hline
\end{tabular}

$\mathrm{A}-$ The $6 \mathrm{w} \mathrm{O}+\mathrm{D}$ control rats exhibit a significant decrease in density of cholinergic fiber endings in the hippocampal region; B - comparison of OD values among the three groups; C - Result of AChE activity detection in the hippocampal region. Comparison with the negative control indicates that $* \mathrm{p}<0.05$.

The conditions of cholinergic fiber endings in the hippocampal region were observed through AChE histochemical staining in combination with OD quantitative analysis. Compared with the rats of the control group and the $3 \mathrm{w} \mathrm{O}+\mathrm{D}$ group, the rats of the $6 \mathrm{w} \mathrm{O}+\mathrm{D}$ group exhibit a significant decrease in the density of cholinergic fiber endings in the hippocampal region (Fig. 2A). There is no significant difference in OD values between the control group rats and the $3 \mathrm{w} \mathrm{O}+\mathrm{D}$ group rats (Fig. 2B). Similar results were also obtained in AChE activity detection in the hippocampal region. AChE activity of the $6 \mathrm{w} \mathrm{O}+\mathrm{D}$ group of rats significantly decreased whereas there was no significant change in AChE activity of rats between the control group and the $3 \mathrm{w} \mathrm{O}+\mathrm{D}$ group (Fig. 2C) (Tab. 2).

\section{Change of GFAP protein and GSH levels of OVX+D-gal rats}

To analyse activation of astrocytes following OVX and injection of D-gal, we detected expression of GFAP in the hippocampal region using the western blotting method. The semi-quantitative analysis results indicate that the levels of GFAP in the hippocampal region of the $3 \mathrm{w}$ and $6 \mathrm{w} \mathrm{O}+\mathrm{D}$ group of rats are significantly increased but there is no statistical difference between the two groups (Fig. 3A). In the hippocampal region, we observed an oxidation resistance of astrocytes through detection of the GSH level. Compared with the Figure 2 group and $3 \mathrm{w} \mathrm{O}+\mathrm{D}$ group, the $\mathrm{GSH}$ level of the $6 \mathrm{w} \mathrm{O}+\mathrm{D}$ group is significantly decreased. The
GSH level of the $3 \mathrm{w} \mathrm{O}+\mathrm{D}$ group is slightly increased and there is no significant difference compared with the control group (Fig. 3B) (Tab. 3).

\section{Morphological changes of astrocytes of $\mathrm{OVX}+D$-gal rats}

Based on an increasing GFAP level, we further observed activation of astrocytes in the hippocampal region through the immunohistochemical staining method. The hippocampal regions of the $3 \mathrm{w}$ and $6 \mathrm{w} \mathrm{O}+\mathrm{D}$ groups exhibit extensive astrocyte activation. Compared with long and thin processes of resting state astrocytes of the control group, two groups of $\mathrm{O}+\mathrm{D}$ activated astrocytes were large. The processes were thickening and hyperchromatic, highly branched; in addition, many GFAP positive astrocytes of the $6 \mathrm{w} \mathrm{O}+\mathrm{D}$ group exhibit degeneration of cell disintegration (Fig. 4 and Tab. 4)

\section{Changes of the ultrastructure of hippocampal regions of $O V X+D$ - gal rats}

We further studied variations on the subcellular fraction level of astrocytes in the hippocampal region. The somas and processes of astrocytes of the rats of the $3 \mathrm{w}$ and $6 \mathrm{wO}+\mathrm{D}$ groups are increased compared with the control group. The astrocytes of some rats of $6 \mathrm{w} \mathrm{O}+\mathrm{D}$ group also exhibit accumulation or deficiency of glycogenosome and glial filament, swelling of mitochondria or degeneration characterized by abnormal distribution. Particularly in the synapses concentrated region, processes of the hydropic degenerated astrocytes are highly swelling, including denatured synaptic structure (Fig. 5 and Tab. 5).

\section{Damage of blood brain barrier of $\mathrm{OVX}+D$-gal rats}

To evaluate changes in the ultrastructure of endothelial cells and abnormalities of endfeet of astrocytes around the blood ves-
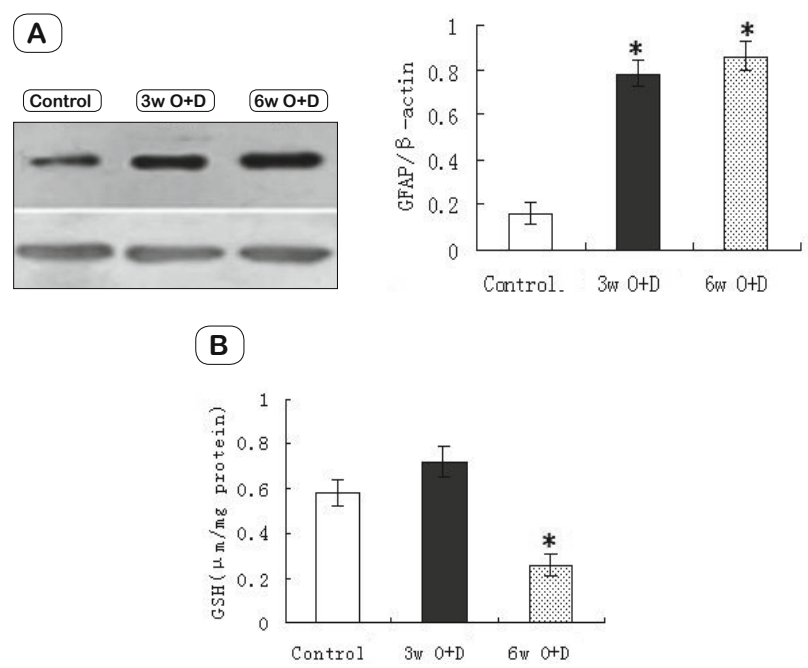

Tab. 3. GFAP protein and GSH levels of $\mathrm{OVX}+\mathrm{D}$-gal rats.

\begin{tabular}{ll}
\hline Control Group & Control Group \\
\hline $3 w \mathrm{O}+\mathrm{D}$ Group & $3 \mathrm{w} \mathrm{O}+\mathrm{D}$ Group \\
\hline 6w O+D Group & $6 \mathrm{w} \mathrm{O}+\mathrm{D}$ Group \\
\hline Comparison with the control group indicates that $\mathrm{p}<0.05$.
\end{tabular}



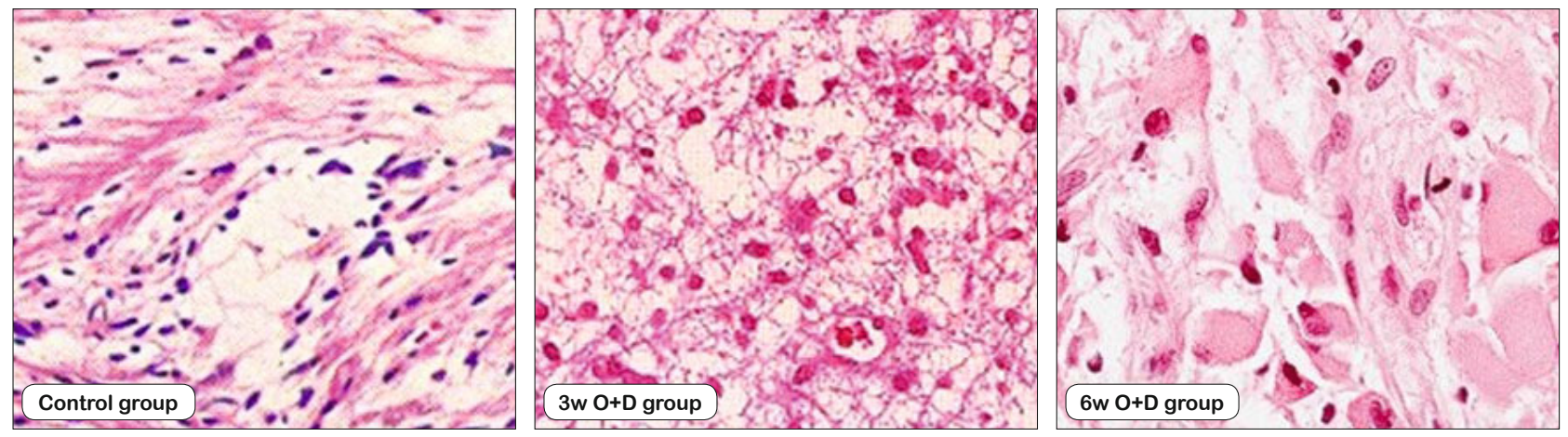

Tab. 4. Morphologic changes of rat astrocytes of each group.

\begin{tabular}{ll}
\hline Control Group & Control Group \\
\hline $3 \mathrm{w}$ O+D Group & $3 \mathrm{w} \mathrm{O+D} \mathrm{Group}$ \\
\hline 6w O+DGroup & $6 \mathrm{w} \mathrm{O+D} \mathrm{Group}$ \\
\hline
\end{tabular}
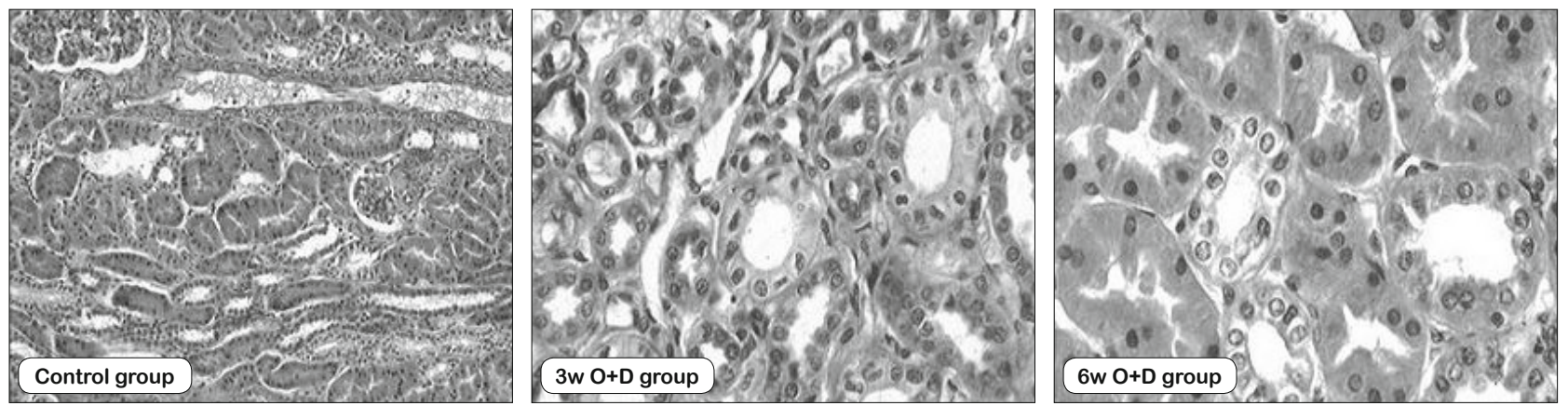

Tab. 5. Changes of star ultrastructures of the hippocampal region observed with an electron microscope.

\begin{tabular}{ll}
\hline Control Group & Control Group \\
\hline 3w O+DGroup & 3 w O+D Group \\
\hline 6 w O+DGroup & 6 w O+D Group \\
\hline
\end{tabular}
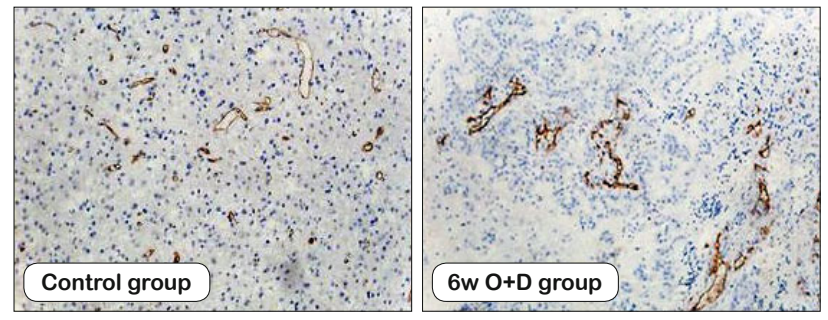

Tab. 6. Permeability variation in blood brain barrier of rats injected with HRP.

\begin{tabular}{ll}
\hline Control Group & Control Group \\
\hline 6w O+DGroup & $6 \mathrm{w} \mathrm{O+D} \mathrm{Group}$ \\
\hline
\end{tabular}

sels, we investigated the permeability of blood brain barrier of the $6 \mathrm{w} \mathrm{O+D}$ group rats by means of intravascular injection of HRP. The results showed that the hippocampus and some regions of the cerebral cortex of the $6 \mathrm{w} \mathrm{O}+\mathrm{D}$ group of rats exhibit leakage of HRP in the vicinity of the blood vessels. However, HRP reaction products of the control group are distributed within the blood vessels and show no signs of significant leakage (Fig. 6 and Tab. 6).

\section{Discussion}

Estrogen deficiency and oxidative stress are two generally accepted risk factors (11). A number of in vitro and in vivo experiments have shown that estrogen can function as an antioxidant to resist oxidative stress thus exhibiting its neuroprotective effect (12). Hence, oxidative stress may aggravate occurrence and progression of $\mathrm{AD}$ under the condition of endogenous estrogen deficiency (13). In the experiment, the rats of the $6 \mathrm{w} \mathrm{O}+\mathrm{D}$ group exhibit progressive cognitive function retrogression and cholinergic loss. Compared with the control group, the rats of the $3 \mathrm{w}$ $\mathrm{O}+\mathrm{D}$ show no signs of significant cognitive function retrogression and cholinergic loss, indicating the cholinergic damage is progressive in the model. Compared to the acute cholinergic damage AD animal model with fornix hippocampus fimbria being transected and the lateral ventricle injected with $\mathrm{A} \beta$ or immunotoxin 192IgGsaporin etc., the model can better simulate the slow pathophysiologic course of AD (14).

In the recent years, more and more evidence has shown that astrocytes can function as target spots for estrogen to exhibit it's neuroprotective effect in the brain (15). Estrogen deprivation and 
oxidative stress can lead to activation of astrocytes. Hence, we infer that astrocytes play an important role in pathophysiologic changes of the AD animal model (16). In the experiment, astrocytes were extensively activated in the hippocampal regions of the rats of the $3 \mathrm{w} \mathrm{O}+\mathrm{D}$ group but there were no signs of $\mathrm{AChE}$ synaptic endings and decrease of activation. Reaction of astrocytes preceding cholinergic loss may be primary rather than secondary. The astrocyte endfeet around the blood capillaries in the brain play an important role in maintaining functions of the blood brain barrier and stability of internal environment, e.g. adjusting nutrient translocation in the brain or protecting neurons against invasion of toxic substances (17). Hence, as an important part of blood brain barrier, astrocytes are the first line of defense against oxygen radicals produced by D-gal in the blood flow (18). In addition, as the main generator for GSH in the brain, astrocytes play their role of antioxidant stress effect by accelerating translocation of dithioglutathione from the brain to the blood flow (19). In general, in the early stages of the AD model, primary activation of astrocytes is favourable. It protects cholinergic neurons confronting oxidative stress mediated cell death by improving GSH level (20). In the early stage of the central nervous system damaged, the activation of astrocytes plays a favourable role for the survival of neurons (21).

In recent years, the relationship between blood-brain barrier damage and $\mathrm{AD}$ occurrence remains controversial but more and more evidence has shown that dysfunction of blood-brain barrier involves or aggravates pathologic changes of degenerative diseases of the nervous system (22-23). Some researchers have demonstrated that in $\mathrm{AD}$ patients or animal models with intravascular diffused soluble $A \beta$, the key to damage of capillaries is the injury of endothelial cells (24). The vascular endothelial cells adjusting macromolecules and passage of circulating cells are the main target spots for oxidative stress aggression (25). In addition, estrogen therapy can alleviate damage of endothelial cells induced by oxidative stress by reducing oxygen radicals generated or regulating the expression of adhesion molecules on endothelial cells (26). Therefore, estrogen deprivation can aggravate damage to endothelial cells by oxidative stress.

So far, a majority of researches have primarily concerned damage or dysfunction of endothelial cells of the blood brain barrier in $\mathrm{AD}$ and observations on changes of astrocytes endfeet in the vicinity of the blood vessels are few (28). In the experiment, we first found coarctation of vascular lumens in the AD model and swelling of astrocytes endfeet in the vicinity of the blood vessels. Astrocytes are closely connected with cerebral capillaries and involved in the composition of blood brain barrier by inducing tight junction of endothelium. Lack of glial intermediate filaments of astrocytes can increase the permeability of blood brain barrier and lead to damage of the neuron microenvironment. This viewpoint has been demonstrated in GFAP knockout mouse and GFAP/protein double knockout mouse (29). In the HRP permeability experiment, the damage of the blood brain barrier completeness may be caused by lesion of endfeet of the astrocytes in the vicinity of the blood vessels in rats of the $6 \mathrm{w} \mathrm{O}+\mathrm{D}$ group.
Tight junction plays an important role in maintaining the completeness of blood brain barrier (30). No extensive HRP leakage exists in the hippocampal regions of rats of the $6 \mathrm{w} \mathrm{O}+\mathrm{D}$ group, which is consistent with tight junction exhibiting no significant changes. Nevertheless, fabrication of this model is a slowly progressive process. Even slight damage of the blood brain barrier can also increase toxic products entering the brain. There is no doubt that the damaged blood brain barrier deteriorates. It is thus clear that damage of blood brain barrier is also involved in the pathogenic process of the AD animal model.

In a word, the model indicates that astrocytes are involed in the process of neuropathological changes. Hence, remodelling of the neuroprotective effects of astrocytes may become a therapeutic target for $\mathrm{AD}$ and some other degenerative diseases of the nervous system.

\section{References}

1. Serrano-Pozo A, Muzikansky A et al. Differential relationships of reactive astrocytes and microglia to fibrillar amyloid deposits in Alzheimer disease. J Neuropathol Exp Neurol 2013; 72 (6): 462-471.

2. Wang G, Dinkins $M$ et al. Astrocytes secrete exosomes enriched with proapoptotic ceramide and prostate apoptosis response 4 (PAR-4): potential mechanism of apoptosis induction in Alzheimer disease. J Biol Chem 2012; 287 (25): 21384-2195.

3. Myung NH, Zhu X et al. Evidence of DNA damage in Alzheimer disease: phosphorylation of histone H2AX in astrocytes. Age (Dordr) 2008; 30 (4): 209-215.

4. Maki RA, Tyurin VA et al. Aberrant expression of myeloperoxidase in astrocytes promotes phospholipid oxidation and memory deficits in a mouse model of Alzheimer disease. J Biol Chem 2009; 284 (5): 3158-3169.

5. Utter S, Tamboli IY et al. Cerebral small vessel disease-induced apolipoprotein E leakage is associated with Alzheimer disease and the accumulation of amyloid beta-protein in perivascular astrocytes. J Neuropathol Exp Neurol 2008; 67 (9): 842-856.

6. Zhu X, Siedlak SL et al. Neuronal binucleation in Alzheimer disease hippocampus. Neuropathol Appl Neurobiol0 2008; 34 (4): 457-465.

7. Isik AT, Bozoglu $\mathbf{E}$ et al. aChE and BuChE inhibition by rivastigmin have no effect on peripheral insulin resistance in elderly patients with Alzheimer disease. J Nutr Health Aging 2012; 16 (2): 139-141.

8. Liu ZB, Niu WM et al. Effect of "Xiusanzhen" on learning-memory ability and hippocampal ChAT and AChE activity in Alzheimer disease rats. Zhen Ci Yan Jiu 2009; 34 (1): 48-51.

9. Kamphuis W, Mamber C et al. GFAP isoforms in adult mouse brain with a focus on neurogenic astrocytes and reactive astrogliosis in mouse models of Alzheimer disease. PLoS One 2012; 7 (8): 823-825.

10. Bartha R, Smith M et al. High field (1)H MRS of the hippocampus after donepezil treatment in Alzheimer disease. Prog Neuropsychopharmacol Biol Psychiatry 2008; 32 (3): 786-793.

11. Ramamoorthy M, Sykora $P$ et al. Sporadic Alzheimer disease fibroblasts display an oxidative stress phenotype. Free Radic Biol Med 2012; 53 (6): 1371-1380.

12. Sultana R, Robinson RA et al. Do proteomics analyses provide insights into reduced oxidative stress in the brain of an Alzheimer disease transgenic mouse model with an M631L amyloid precursor protein sub- 


\section{2-698}

stitution and thereby the importance of amyloid-beta-resident methionine 35 in Alzheimer disease pathogenesis?. Antioxid Redox Signal 2012; 17 (11): $1507-1514$.

13. Perry G, Phelix CF et al. Untangling the vascular web from Alzheimer disease and oxidative stress. Can J Neurol 2012; 39 (1): 4-6.

14. Castellani RJ, Moreira PI et al. The role of iron as a mediator of oxidative stress in Alzheimer disease. Biofactors 2012; 38 (2): 133-138.)

15. Penner J, Rupsingh R et al. Increased glutamate in the hippocampus after galantamine treatment for Alzheimer disease. Prog Neuropsychopharmacol Biol Psychiatry 2010; 34 (1): 104-110.

16. Zampagni M, Wright $\mathbf{D}$ et al. Novel S-acyl glutathione derivatives prevent amyloid oxidative stress and cholinergic dysfunction in Alzheimer disease models. Free Radic Biol Med 2012; 52 (8): 1362-1371.

17. Owen JB, Sultana R et al. Oxidative modification to LDL receptorrelated protein 1 in hippocampus from subjects with Alzheimer disease: implications for Abeta accumulation in AD brain. Free Radic Biol Med 2010; 49 (11): 1798-1803.

18. Clark TA, Lee HP et al. Oxidative Stress and its Implications for Future Treatments and Management of Alzheimer Disease. Int J Biomed 2010; 6 (3): 225-227.

19. Kuzyk A, Kastyak M et al. Association among amyloid plaque, lipid, and creatine in hippocampus of TgCRND8 mouse model for Alzheimer disease. J Biol Chem 2010; 285 (41): 31202-31207.

20. Lee HP, Pancholi $\mathbf{N}$ et al. Early induction of oxidative stress in mouse model of Alzheimer disease with reduced mitochondrial superoxide dismutase activity. PLoS One 2012; 7 (1): 33-34.

21. Hong Y J, Yoon B et al. Differences in microstructural alterations of the hippocampus in Alzheimer disease and idiopathic normal pressure hydrocephalus: a diffusion tensor imaging study. AJNR Am J Neuroradiol 2010; 31 (10): 1867-1872.
22. Zhu SX, Sun GJ et al. Effects of electroacupuncture on learning and memory ability and glial cells of the hippocampus in the rat of Alzheimer disease. Zhongguo Zhen Jiu 2009; 29 (2): 133-136.

23. Barone E, Cenini G et al. Long-term high-dose atorvastatin decreases brain oxidative and nitrosative stress in a preclinical model of Alzheimer disease: a novel mechanism of action. Pharmacol Res 2011; 63 (3): 172-180.

24. Wang Z, Zhao $\mathbf{C}$ et al. Regional metabolic changes in the hippocampus and posterior cingulate area detected with 3-Tesla magnetic resonance spectroscopy in patients with mild cognitive impairment and Alzheimer disease. Acta Radiol 2009; 50 (3): 312-319.

25. Butterfield DA. Oxidative stress in Alzheimer disease: synergy between the Butterfield and Markesbery laboratories. Neuromolecular Med 2011; 13 (1): 19-22.

26. Satoh J, Tabunoki H et al. Molecular network analysis suggests aberrant CREB-mediated gene regulation in the Alzheimer disease hippocampus. Dis Markers 2009; 27 (5): 239-252.

27. Bonda DJ, Wang $X$ et al. Oxidative stress in Alzheimer disease: a possibility for prevention. Neuropharmacology 2010; 59 (4): 290-294.

28. Takeda S, Sato $\mathbf{N}$ et al. Increased blood-brain barrier vulnerability to systemic inflammation in an Alzheimer disease mouse model. Neurobiol Aging 2013; 34 (8): 2064-2070.

29. Aluise CD, Robinson RA et al. Preclinical Alzheimer disease: brain oxidative stress, Abeta peptide and proteomics. Neurobiol Dis 2010; 39 (2): 221-228.

30. Liu H, Funke SA et al. Transport of Alzheimer disease amyloid-betabinding D-amino acid peptides across an in vitro blood-brain barrier model. Rejuvenation Res 2010; 13 (2): 210-213. 